\title{
A New Variant of the Cramer-Shoup KEM Secure against Chosen Ciphertext Attack
}

\author{
Joonsang Baek ${ }^{1}$ \\ Willy Susilo ${ }^{2}$ \\ Joseph K. Liu ${ }^{1}$ \\ Jianying Zhou ${ }^{1}$ \\ ${ }^{1}$ Institute for Infocomm Research, Singapore \\ ${ }^{2}$ University of Wollongong, Australia
}

March 25, 2009

\begin{abstract}
We propose a new variant of the Cramer-Shoup KEM (key encapsulation mechanism). The proposed variant is more efficient than the original Cramer-Shoup KEM scheme in terms of public key size and encapsulation cost, but is proven to be (still) secure against chosen ciphertext attack in the standard model, relative to the Decisional Diffie-Hellman problem.
\end{abstract}

\section{Introduction}

Motivation. At Crypto '98, Cramer and Shoup [9] proposed the first practical public key encryption (PKE) scheme whose security against adaptive chosen ciphertext attack (which we simply call "CCA" throughout this paper) can be proven without depending on the random oracle model [6]. This is a striking result as the chosen ciphertext security without random oracles could be achieved by only adding a few more exponentiations to the original ElGamal encryption scheme, in contrast to the computationally heavy solutions $[11,20]$ based on zero-knowledge proofs proposed before. Nearly seven years later, a major improvement on the performance of the Cramer-Shoup PKE scheme was made by Kurosawa and Desmedt [17]. They were able to obtain a very efficient hybrid PKE scheme by simplifying the CramerShoup PKE scheme with the help of the "ciphertext authenticity checking" mechanism of the underlying symmetric encryption primitive. Afterwards, Hofheinz and Kiltz [14] came up with a dual version of the Kurosawa-Desmedt PKE scheme. Note that chosen ciphertext security of all these schemes are relative to the (standard) Decisional Diffie-Hellman (DDH) problem.

In the full version of their Crypto '98 paper, Cramer and Shoup [10] formulated a framework called "KEM/DEM (Key Encapsulation Mechanism/Data Encapsulation Mechanism)". A KEM is a public key scheme that outputs a (session) key taking public key as input. According to the KEM/DEM framework, a (hybrid) PKE scheme secure against CCA can be constructed in such a way that a key output by a CCA-secure KEM scheme ${ }^{1}$ is used as a ses-

\footnotetext{
${ }^{1}$ The CCA security notion for KEM will be defined in Section 2.
} 
sion key for an one-time CCA-secure DEM (i.e., symmetric encryption) scheme that encrypts a plaintext message.

In the same paper, Cramer and Shoup proposed a KEM scheme based on their original PKE scheme, which we denote by "CS-KEM", and showed it is CCA-secure assuming that the DDH problem is hard. Interestingly, however, it was shown [13] that the KEM scheme extracted from Kurosawa and Desmedt's hybrid PKE scheme, which we denote by "KDKEM", does not satisfy full CCA-security even though the hybrid PKE scheme remains secure against CCA. Abe et al. [1] showed later that the KD-KEM scheme is actually secure against "LCCA (predicate-dependent CCA)" which is weaker than usual CCA-security of KEM. Similarly, the KEM scheme extracted from Hofheinz and Kiltz's [14] hybrid PKE scheme, denoted "HK-KEM", was shown to be secure against CCCA (constrained CCA), which is also weaker than the usual CCA-security of KEM.

Hence, the CS-KEM scheme is, though less efficient than the KD-KEM and HK-KEM schemes, the only KEM scheme that is fully CCA-secure without random oracles, assuming that the DDH problem is hard. A remaining question is whether the performance of the CS-KEM scheme can be further improved. In this paper, we give a positive answer to this question.

Recent Developments. In 2007, Kiltz [16] proposed a KEM scheme whose CCA security is based on the gap hashed Diffie-Hellman problem. An interesting feature of this scheme is that different from the CS-KEM scheme, a key can be computed from one of the public key components used to create one ciphertext component. More precisely, let $p k=(q, g, c, d)$ be public key, where $g$ is a generator of a group of prime order $q$; $c=g^{x}$ and $d=g^{y}$ for some random $(x, y) \in \mathbb{Z}_{q}^{*}$. In this scheme, a ciphertext and its corresponding key is computed as $\left(g^{r},\left(c^{\alpha} d\right)^{r}\right)$ and $\operatorname{KDF}\left(c^{r}\right)$ respectively, where KDF denotes a key derivation function. As mentioned earlier, the public $c$ used to create $\left(c^{\alpha} d\right)^{r}$ is reused to produce $c^{r}$. Note here that one cannot expect a computational gain even if $c$ is reused. However, if $d$ were reused, a computational cost could be reduced by computing $c^{r \alpha}$ and $d^{r}$ separately to generate $\left(c^{\alpha} d\right)^{r}$ and using $d^{r}$ as a key. Indeed, Lu et al. [18] recently showed that this modified version of Kiltz's KEM scheme is CCA-secure.

More recently, as one of the applications of their new computational problem called "Twin Diffie-Hellman", Cash et al. [8] proposed a new variant of Cramer and Shoup's PKE scheme and showed that it is CCA-secure under the hashed decisional Diffie-Hellman assumption, which is weaker than the usual DDH assumption ${ }^{2}$. Although this variant has interesting theoretical implications, it is computationally more expensive than the original Cramer and Shoup's one and hence ours.

Our Contributions. We observe that it is also possible to apply the structure of Kiltz's KEM scheme to the CS-KEM scheme. As a result, we could construct a KEM scheme which is proven to be fully CCA-secure without random oracles assuming that the DDH problem is hard, while it is more efficient than the CS-KEM scheme. The crux is the efficiency of our scheme in terms of a shorter public/private key pair and improved encapsulation speed. However, we honestly state that the improvement on the encapsulation speed would not be very much dramatic due to the advancement of fast multi-exponentiation algorithms

\footnotetext{
${ }^{2}$ Note that although [8] focuses only on a PKE scheme, a corresponding KEM scheme can easily be derived and analyzed in an obvious way.
} 
$[2,7,19]$, which makes the cost for computing double exponentiation very close to the cost of computing a single exponentiation. Nevertheless, the proposed scheme has a new structure, which reduces one group element of the public key of the CS-KEM scheme. We believe it is also theoretically interesting in that it shows yet another way of constructing a more efficient variant of the CS-KEM without sacrificing full CCA-security.

\section{Preliminaries}

In this section, we review the formal notion of key encapsulation mechanism (KEM) and its security against adaptive chosen ciphertext attack (CCA). We also review building blocks used in our construction of KEM which will be presented in Section 3.

Key Encapsulation Mechanism (KEM). The KEM scheme, denoted KEM, consists of the following algorithms $[10,22,15]$.

- Key Generation: Taking $1^{\lambda}$ for a security parameter $\lambda \in \mathbb{Z}_{\geq 0}$ as input, this algorithm generates a public/private key pair $(p k, s k)$.

- Encapsulation: Taking $1^{\lambda}$ and a public key $p k$ as input, this algorithm generates a ciphertext/(symmetric) key pair $(\psi, K)$.

- Decapsulation: Taking $1^{\lambda}$, a private key $s k$ and a ciphertext $\psi$ as input, this algorithm outputs either a (symmetric) key $K$ or the special symbol $\perp$, meaning "reject".

The security against CCA of KEM is defined as follows. Consider any attacker $\mathcal{A}$ and any value $\lambda>0$ for security parameter in the following game $\operatorname{GameCCA}_{\mathcal{A}}^{\mathrm{KEM}}(\lambda)$ in which $\mathcal{A}$ interacts with the challenger.

Phase 1: The challenger runs the key generation algorithm providing $1^{\lambda}$ as input to generate a public/private key pair $(p k, s k)$. The challenger then computes a challenge ciphertext $\phi^{*}$ and a key $K_{1}^{*}$ by running the encapsulation algorithm. It also picks $K_{0}^{*} \in S_{K}$ at random, where $S_{K}$ denotes the key space. It then picks $\beta \in\{0,1\}$ at random and gives $\left(p k, \phi^{*}, K_{\beta}^{*}\right)$ to $\mathcal{A}$.

Phase 2: $\mathcal{A}$ submits ciphertexts, each of which is denoted by $\phi$. On receiving $\phi$, the challenger runs the decapsulation algorithm on input $\phi$ and passes the resulting decapsulation to $\mathcal{A}$. At the end of this phase, $\mathcal{A}$ outputs its guess $\beta^{\prime} \in\{0,1\}$.

We define the output of the game to be 1 if $\beta^{\prime}=\beta$, and 0 otherwise. $\mathcal{A}$ 's success is defined by the probability

$$
\operatorname{Adv}_{\mathcal{A}, \mathrm{KEM}}^{\mathrm{CCA}}(\lambda)=\left|\operatorname{Pr}\left[\operatorname{GameCCA}_{\mathcal{A}}^{\mathrm{KEM}}(\lambda)=1\right]-\frac{1}{2}\right| .
$$

We say that $\mathrm{KEM}$ is CCA-secure if $\operatorname{Adv}_{\mathrm{KEM}}^{\mathrm{CCA}}(\lambda)=\max _{\mathcal{A}}\left\{\operatorname{Adv}_{\mathcal{A}, \mathrm{KEM}}^{\mathrm{CCA}}(\lambda)\right\}$ is negligible for any attacker $\mathcal{A}$. 
The Decisional Diffie-Hellman Problem. We now review the definition of the Decisional DiffieHellman $(\mathrm{DDH})$ problem. Let $\mathcal{D}$ be an attacker. Let $\mathbb{G}$ be a finite cyclic group generated by $g \in \mathbb{G}$. Let $q$ be a prime order of $\mathbb{G}$, whose size depends on the security parameter $\lambda$. We define the DDH problem using the attacker $\mathcal{D}$ 's advantage in distinguishing two distributions:

$$
\begin{aligned}
\operatorname{Adv}_{\mathcal{D}, \mathbb{G}}^{\mathrm{DDH}}(\lambda) & =\mid \operatorname{Pr}\left[a \stackrel{\mathrm{R}}{\leftarrow} \mathbb{Z}_{q} ; b \stackrel{\mathrm{R}}{\leftarrow} \mathbb{Z}_{q}: 1 \leftarrow \mathcal{D}\left(1^{\lambda}, g^{a}, g^{b}, g^{a b}\right)\right] \\
& -\operatorname{Pr}\left[a \stackrel{\mathrm{R}}{\leftarrow} \mathbb{Z}_{q} ; b \stackrel{\mathrm{R}}{\leftarrow} \mathbb{Z}_{q} ; r \stackrel{\mathrm{R}}{\leftarrow} \mathbb{Z}_{q}: 1 \leftarrow \mathcal{D}\left(1^{\lambda}, g^{a}, g^{b}, g^{r}\right)\right] \mid .
\end{aligned}
$$

Equivalently $[9,10,12]$,

$$
\begin{aligned}
\operatorname{Adv}_{\mathcal{D}, \mathbb{G}}^{\mathrm{DDH}}(\lambda) & =\mid \operatorname{Pr}\left[w \stackrel{\mathrm{R}}{\leftarrow} \mathbb{Z}_{q} ; g_{2} \leftarrow g_{1}^{w} ; r \stackrel{\mathrm{R}}{\leftarrow} \mathbb{Z}_{q}: 1 \leftarrow \mathcal{D}\left(1^{\lambda}, g_{1}, g_{2}, g_{1}^{r}, g_{2}^{r}\right)\right] \\
& -\operatorname{Pr}\left[w \stackrel{\mathrm{R}}{\leftarrow} \mathbb{Z}_{q} ; g_{2} \leftarrow g_{1}^{w} ; r^{\prime} \stackrel{\mathrm{R}}{\leftarrow} \mathbb{Z}_{q} \backslash\{r\}: 1 \leftarrow \mathcal{D}\left(1^{\lambda}, g_{1}, g_{2}, g_{1}^{r}, g_{2}^{r^{\prime}}\right)\right] \mid,
\end{aligned}
$$

where $g_{1}$ is the generator of $\mathbb{G}$.

We say that the DDH problem is hard if $\mathbf{A d v}_{\mathbb{G}}^{\mathrm{DDH}}(\lambda)=\max _{\mathcal{D}}\left\{\mathbf{A d v}_{\mathcal{D}, \mathbb{G}}^{\mathrm{CCA}}(\lambda)\right\}$ is negligible for any attacker $\mathcal{D}$.

Target Collision Resistant Hash Function (TCR). The security of the target collision resistant hash function denoted by $\mathrm{H}$ is defined as follows. Given a $n$ tuple of group elements $x \in \mathbb{G}^{n}$, it is hard for an attacker $\mathcal{B}_{1}$ to find $y \neq x$ such that $\mathrm{H}(x)=\mathrm{H}(y)$. We define the attacker $\mathcal{B}_{1}$ 's success probability by $\mathbf{A d v}_{\mathcal{B}_{1}, \mathrm{H}}^{\mathrm{COL}}(\lambda)$. We say that $\mathrm{H}$ is target collision-resistant if $\operatorname{Adv}_{\mathrm{H}}^{\mathrm{COL}}(\lambda)=$ $\max _{\mathcal{B}_{1}}\left\{\mathbf{A d v}_{\mathcal{B}_{1}, \mathrm{H}}^{\mathrm{COL}}(\lambda)\right\}$ is negligible for any attacker $\mathcal{B}_{1}$.

Key Derivation Function (KDF). In the proposed variant of the KD-KEM scheme, we will use the key derivation function denoted by KDF. Specifically, KDF takes two random elements $a$ and $b$ in the group $\mathbb{G}$ as input. Let $l$ be the length of the output of KDF, which depends on the security parameter $\lambda$. We define the security of KDF with respect to an attacker $\mathcal{B}_{2}$ as follows. (Below, "ROR" stands for "real or random".)

$$
\begin{aligned}
\operatorname{Adv}_{\mathcal{B}_{2}, \mathrm{KDF}}^{\mathrm{ROR}}(\lambda) & =\mid \operatorname{Pr}\left[a, b \stackrel{\mathrm{R}}{\leftarrow} \mathbb{G}: 1 \leftarrow \mathcal{B}_{2}\left(1^{\lambda}, a, \operatorname{KDF}(a, b)\right)\right] \\
& -\operatorname{Pr}\left[a \stackrel{\mathrm{R}}{\leftarrow} \mathbb{G} ; \mu \stackrel{\mathrm{R}}{\leftarrow}\{0,1\}^{l}: 1 \leftarrow \mathcal{B}_{2}\left(1^{\lambda}, a, \mu\right)\right] \mid .
\end{aligned}
$$

We say that KDF is secure if $\operatorname{Adv}_{\mathrm{KDF}}^{\mathrm{ROR}}(\lambda)=\max _{\mathcal{B}_{2}}\left\{\mathbf{A d v}_{\mathcal{B}_{2}, \mathrm{KDF}}^{\mathrm{ROR}}(\lambda)\right\}$ is negligible for any attacker $\mathcal{B}_{2}$.

Notice that the above security requirement on KDF is the same as that of the KDF functions used in [10].

\section{The Proposed Variant of the Cramer-Shoup KEM}

Description. We describe our variant of the CS-KEM scheme, which we denote by "П", as follows. (Readers are referred to the end of Section 1 for the underlying idea of our scheme.)

Key Generation: Pick a group $\mathbb{G}$ of prime order $q$ and generators $g_{1}$ and $g_{2}$ of $\mathbb{G}$. Pick a target-collision resistant hash function $\mathrm{H}:\{0,1\}^{*} \rightarrow \mathbb{Z}_{q}^{*}$ and a key derivation function KDF. Then choose $\left(x_{1}, x_{2}, y_{1}, y_{2}\right) \in \mathbb{Z}_{q}^{4}$ at random and compute

$$
c=g_{1}^{x_{1}} g_{2}^{x_{2}}, d=g_{1}^{y_{1}} g_{2}^{y_{2}} .
$$

Return public key $p k=\left(\mathbb{G}, q, g_{1}, g_{2}, c, d, \mathrm{H}, \mathrm{KDF}\right)$ and private key $s k=\left(p k, x_{1}, x_{2}, y_{1}, y_{2}\right)$. 
Encapsulation: Pick $r \in \mathbb{Z}_{q}^{*}$ at random and compute

$$
u_{1}=g_{1}^{r}, u_{2}=g_{2}^{r}, \alpha=\mathrm{H}\left(u_{1}, u_{2}\right), v=c^{r} d^{r \alpha}, K=\operatorname{KDF}\left(u_{1}, c^{r}\right) .
$$

Return a ciphertext $\psi=\left(u_{1}, u_{2}, v\right)$ and a key $K$.

Decapsulation: Upon receiving $\psi=\left(u_{1}, u_{2}, v\right)$, compute

$$
\alpha=\mathrm{H}\left(u_{1}, u_{2}\right), v^{\prime}=u_{1}^{x_{1}+y_{1} \alpha} u_{2}^{x_{2}+y_{2} \alpha}, K=\operatorname{KDF}\left(u_{1}, u_{1}^{x_{1}} u_{2}^{x_{2}}\right)
$$

If $v^{\prime}=v$ then return $K$; otherwise, return $\perp$.

We show that the scheme $\Pi$ is CCA-secure, relative to the DDH problem. More precisely, we prove the following theorem.

Theorem 1 The KEM scheme $\Pi$ is CCA-secure assuming that the DDH problem is hard and the underlying hash function $\mathrm{H} s$ target collision-resistant and key derivation function KDF is secure. More precisely, we have

$$
\mathbf{A d v}_{\Pi}^{C C A}(\lambda) \leq \mathbf{A d v}_{\mathbb{G}}^{D D H}(\lambda)+\mathbf{A d v}_{\mathrm{H}}^{C O L}(\lambda)+\mathbf{A d v}_{\mathrm{KDF}}^{R O R}(\lambda)+\frac{q_{D}}{q} .
$$

where $\lambda$ denotes the security parameter and $q_{D}$ is the number of queries to the decapsulation oracle.

Outline of Proof. The basic idea of the proof essentially follows the logic of the proofs of the CS-KEM [10] and CS-PKE [9] schemes. We need to show that by using a CCA-attacker for the scheme $\Pi$ as a subroutine, a DDH attacker can solve the DDH problem: When the DDH attacker is given a right Diffie-Hellman tuple $\left(g_{1}, g_{2}, g_{1}^{r}, g_{2}^{r}\right)$, it can perfectly simulate the environment of the CCA-attacker. On the other hand, when it is given $\left(g_{1}, g_{2}, g_{1}^{r}, g_{2}^{r^{\prime}}\right)$ where $r^{\prime} \neq r$, the output of the decapsulation oracle will not be legitimate but we will show that this one won't be a problem.

In our proof, there is an important difference from the proofs of the CS-KEM/CS-PKE schemes. Since the public key component $c$ used to create $v=c^{r} d^{r \alpha}$ is "reused" to produce a key material $c^{r}$, we need to assume that the attacker's view include $c, d, v$ and $c^{r}$ when breaking the confidentiality (i.e. "key indistinguishability") of the scheme $\Pi$. (Note that this is different from the CS-KEM/CS-PKE schemes in which an independent public key component is used to produce a key.) By using an argument from linear algebra, we show that fortunately, this does not cause a problem. (In particular, readers are referred to Equation $(12))$.

Proof. Fix an attacker $\mathcal{A}$ that breaks CCA-security of the scheme $\Pi$. Also, fix an attacker $\mathcal{D}$ that is to solve the DDH problem.

Simulation. The DDH attacker $\mathcal{D}$ simulates the environment of $\mathcal{A}$ as follows. Assume that $\mathcal{D}$ is given a DDH instance $\left(g_{1}, g_{2}, u_{1}, u_{2}\right)$ where $g_{1}$ and $g_{2}$ are generators of a group $\mathbb{G}$ of prime-order $q$. $\mathcal{D}$ chooses $\left(x_{1}, x_{2}, y_{1}, y_{2}\right) \in \mathbb{Z}_{q}^{4}$ at random and computes $c=g_{1}^{x_{1}} g_{2}^{x_{2}}$ and $d=g_{1}^{y_{1}} g_{2}^{y_{2}}$. $\mathcal{D}$ also chooses a hash function $\mathrm{H}$ and a key derivation function KDF, and gives $p k=\left(\mathbb{G}, q, g_{1}, g_{2}, c, d, \mathrm{H}, \mathrm{KDF}\right)$ as a public key to $\mathcal{A}$. 
When $\mathcal{A}$ queries ciphertexts to the decapsulation oracle in the find stage, $\mathcal{D}$ decapsulates them using $\left(x_{1}, x_{2}, y_{1}, y_{2}\right)$.

$\mathcal{D}$ simulates the challenge ciphertext and the key as follows. $\mathcal{D}$ first sets $u_{1}^{*}=u_{1}$ and $u_{2}^{*}=$ $u_{2}$, and computes $\alpha^{*}=\mathrm{H}\left(u_{1}^{*}, u_{2}^{*}\right), v^{*}=\left(u_{1}^{*}\right)^{x_{1}+y_{1} \alpha^{*}}\left(u_{2}^{*}\right)^{x_{2}+y_{2} \alpha^{*}}$ and $K_{1}^{*}=\operatorname{KDF}\left(u_{1}^{*},\left(u_{1}^{*}\right)^{x_{1}}\left(u_{2}^{*}\right)^{x_{2}}\right)$. $\mathcal{D}$ also chooses $K_{0}^{*}$ at random from the output space of KDF and picks $\beta \in\{0,1\}$ at random. $\mathcal{D}$ finally gives $\mathcal{A}$ the challenge ciphertext-key pair $\left(\psi^{*}, K_{\beta}^{*}\right)$ where $\psi^{*}=\left(u_{1}^{*}, u_{2}^{*}, v^{*}\right)$.

When $\mathcal{A}$ queries ciphertexts, all of which are different from $\psi^{*}$, to the decapsulation oracle in the find stage, $\mathcal{D}$ decapsulates them using $\left(x_{1}, x_{2}, y_{1}, y_{2}\right)$.

Finally, when $\mathcal{A}$ outputs its guess $\beta^{\prime}, \mathcal{D}$ outputs 1 if $\beta^{\prime}=\beta$; otherwise, outputs 0 .

Analysis. We first analyze the case when $\mathcal{D}$ is given $\left(g_{1}, g_{2}, g_{1}^{r^{*}}, g_{2}^{r^{*}}\right)$. First, we prove the following lemma.

\section{Lemma 1}

$$
\operatorname{Pr}\left[\mathcal{D}\left(1^{\lambda}, g_{1}, g_{2}, g_{1}^{r^{*}}, g_{2}^{r^{*}}\right)=1\right]=\operatorname{Pr}\left[\operatorname{GameCCA}_{\mathcal{A}}^{\Pi}(\lambda)=1\right]
$$

Proof. Note that since $\left(x_{1}, x_{2}, y_{1}, y_{2}\right)$ is randomly chosen from $\mathbb{Z}_{q}^{4}$, the public key $p k$ is distributed the same as the public key in the real attack.

By the simulation of the challenge ciphertext presented above, we have

$$
\psi^{*}=\left(u_{1}^{*}, u_{2}^{*}, v^{*}\right)=\left(g_{1}^{r^{*}}, g_{2}^{r^{*}},\left(g_{1}^{r^{*}}\right)^{x_{1}+y_{1} \alpha^{*}}\left(g_{2}^{r^{*}}\right)^{x_{2}+y_{2} \alpha^{*}}\right)=\left(g_{1}^{r^{*}}, g_{2}^{r^{*}}, c^{r^{*}} d^{r^{*} \alpha^{*}}\right)
$$

and

$$
K_{1}^{*}=\operatorname{KDF}\left(u_{1}^{*},\left(g_{1}^{r^{*}}\right)^{x_{1}}\left(g_{2}^{r^{*}}\right)^{x_{2}}\right)=\operatorname{KDF}\left(u_{1}^{*}, c^{r^{*}}\right) .
$$

Since $K_{0}^{*}$ is drawn uniformly at random from the output space of $\mathrm{KDF},\left(\psi^{*}, K_{\beta}^{*}\right)$ has the right distribution.

It remains to show that the output of the decapsulation oracle (both in the simulation and the real attack) has the right distribution. Now, we call a ciphertext $\psi=\left(u_{1}, u_{2}, v\right)$ is invalid if $\log _{g_{1}} u_{1} \neq \log _{g_{2}} u_{2}$. We show that invalid ciphertexts are rejected except for negligible probability.

First, by the public key $p k$ that $\mathcal{A}$ sees, we have the following equations:

$$
\log _{g_{1}} c=x_{1}+x_{2} w
$$

and

$$
\log _{g_{1}} d=y_{1}+y_{2} w
$$

where $w=\log _{g_{1}} g_{2}$. Hence, one can view $\left(x_{1}, x_{2}, y_{1}, y_{2}\right)$ as a random point on the plane defined by (2) and (3). From the challenge ciphertext, we have

$$
\log _{g_{1}} v^{*}=r^{*}\left(x_{1}+x_{2} w+y_{1} \alpha^{*}+y_{2} w \alpha^{*}\right),
$$

where $r^{*}=\log _{g_{1}} u_{1}^{*}=\log _{g_{2}} u_{2}^{*}$ and $\alpha^{*}=\mathrm{H}\left(u_{1}^{*}, u_{2}^{*}\right)$. Note that the challenge ciphertext (whether it is in the simulation or real attack) does not constrain $\left(x_{1}, x_{2}, y_{1}, y_{2}\right)$ as the hyperplane defined by (4) contains the plane formed by the equations (2) and (3). Now consider the following equation obtained from the invalid ciphertext $\psi$ :

$$
\log _{g_{1}} v=r_{1} x_{1}+r_{2} x_{2} w+r_{1} y_{1} \alpha+r_{2} y_{2} w \alpha
$$


where $r_{1}=\log _{g_{1}} u_{1}$ and $r_{2}=\log _{g_{1}} u_{2}$ such that $r_{1} \neq r_{2}$. If the decapsulation oracle does not reject $\psi$, the point $\left(x_{1}, x_{2}, y_{1}, y_{2}\right)$ should lie on the hyperplane defined by (5). But observe that the equations (2), (3) and (5) are linearly independent, so the hyperplane defined by (5) intersects the plane formed by the equations (2) and (3) at a line. This happens with probability $1 / q$, which is negligible.

We now analyze the case when $\mathcal{D}$ is given $\left(g_{1}, g_{2}, g_{1}^{r_{1}^{*}}, g_{2}^{r_{2}^{*}}\right)$ where $r_{1}^{*} \neq r_{2}^{*}$. More precisely, we prove the following lemma.

\section{Lemma 2}

$$
\operatorname{Pr}\left[\mathcal{D}\left(1^{\lambda}, g_{1}, g_{2}, g_{1}^{r_{1}^{*}}, g_{2}^{r_{2}^{*}}\right)=1\right] \leq \frac{1}{2}+\mathbf{A d v}_{\mathrm{KDF}}^{R O R}(\lambda)+\mathbf{A d v}_{\mathrm{H}}^{C O L}(\lambda)+\frac{q_{D}}{q} .
$$

The above bound (6) can be obtained by proving the following Claim 1 and Claim 2.

Recall that if a ciphertext $\psi=\left(u_{1}, u_{2}, v\right)$ is "invalid" then $\log _{g_{1}} u_{1} \neq \log _{g_{2}} u_{2}$. We first prove the following claim.

Claim 1 Let RejlnvC to be an event that the decapsulation oracle rejects all invalid ciphertexts. Then we have

$$
\operatorname{Pr}\left[\beta^{\prime}=\beta \mid \operatorname{RejlnvC}\right] \leq \frac{1}{2}+\mathbf{A d v}_{\mathrm{KDF}}^{R O R}(\lambda)
$$

Proof of Claim 1. First, assume that that the decapsulation oracle rejects all invalid ciphertexts. We consider the distribution of the point $\left(x_{1}, x_{2}, y_{1}, y_{2}\right) \in \mathbb{Z}_{q}^{4}$ conditioned on $\mathcal{A}$ 's view. Since the decapsulation oracle decapsulates only valid ciphertexts by the assumption (the decapsulation oracle rejects all invalid ciphertexts), for each ciphertext $\left(u_{1}, u_{2}, v\right), \mathcal{A}$ gets only linearly dependent relations

$$
\log _{g_{1}} v=r\left(x_{1}+x_{2} w+y_{1} \alpha+y_{2} w \alpha\right),
$$

and

$$
\log _{g_{1}} u_{1}^{x_{1}} u_{2}^{x_{2}}=r\left(x_{1}+x_{2} w\right)
$$

where $r=\log _{g_{1}} u_{1}=\log _{g_{2}} u_{2}$ and $\alpha=\mathrm{H}\left(u_{1}, u_{2}\right)$. (In fact, $\mathcal{A}$ only gets the key which is the output of KDF which "wraps" the key material $u_{1}^{x_{1}} u_{2}^{x_{2}}$.) Hence, no information about the point $\left(x_{1}, x_{2}, y_{1}, y_{2}\right)$ is leaked from querying valid ciphertexts to the decapsulation oracle.

Now consider the challenge ciphertext $\psi^{*}=\left(u_{1}^{*}, u_{2}^{*}, v^{*}\right)$ and the key $K_{1}^{*}=\operatorname{KDF}\left(u_{1}^{*},\left(u_{1}^{*}\right)^{x_{1}}\left(u_{2}^{*}\right)^{x_{2}}\right)$, produced by the simulation. Suppose that $\mathcal{A}$ gets the key material $\left(u_{1}^{*}\right)^{x_{1}}\left(u_{2}^{*}\right)^{x_{2}}$ at the worst case. Since $v^{*}$ and $\left(u_{1}^{*}\right)^{x_{1}}\left(u_{2}^{*}\right)^{x_{2}}$ are in $\mathcal{A}$ 's view, $\left(x_{1}, x_{2}, y_{1}, y_{2}\right)$ should then satisfy the following equations

$$
\log _{g_{1}} v^{*}=r_{1}^{*} x_{1}+r_{2}^{*} x_{2} w+r_{1}^{*} y_{1} \alpha^{*}+r_{2}^{*} y_{2} w \alpha^{*},
$$

where $r_{1}^{*}=\log _{g_{1}} u_{1}^{*}, r_{2}^{*}=\log _{g_{2}} u_{2}^{*}$ with $r_{1}^{*} \neq r_{2}^{*}$ and $\alpha^{*}=\mathrm{H}\left(u_{1}^{*}, u_{2}^{*}\right)$, and

$$
\log _{g_{1}}\left(u_{1}^{*}\right)^{x_{1}}\left(u_{2}^{*}\right)^{x_{2}}=r_{1}^{*} x_{1}+r_{2}^{*} x_{2} w .
$$


Now observe that

$$
\operatorname{det}\left[\begin{array}{cccc}
1 & w & 0 & 0 \\
0 & 0 & 1 & w \\
r_{1}^{*} & r_{2}^{*} w & r_{1}^{*} \alpha^{*} & r_{2}^{*} \alpha^{*} w \\
r_{1}^{*} & r_{2}^{*} w & 0 & 0
\end{array}\right]=w^{2} \alpha^{*}\left(r_{1}^{*}-r_{2}^{*}\right)^{2} \neq 0 .
$$

Hence, the equations $(2),(3),(10)$ and (11) are linearly independent. Note that $\left(u_{1}^{*}\right)^{x_{1}}\left(u_{2}^{*}\right)^{x_{2}}$ is distributed uniformly in $\mathbb{G}$ since $r_{1}^{*}$ and $r_{2}^{*}$ are chosen uniformly at random from $\mathbb{Z}_{q}$ and that $K_{0}^{*}$ has been chosen uniformly at random and independently from anything else. Thus the distribution of $\beta$ is independent from $\mathcal{A}$ 's view under the assumption that KDF is secure and we get the bound (7). This is the end of proof of Claim 1.

We now show that the probability that the decapsulation oracle does not reject all invalid ciphertexts, i.e. $\operatorname{Pr}[\neg$ RejlnvC $]$, is bounded by insecurity of hash function and some negligible probability. Precisely we prove the following claim.

\section{Claim 2}

$$
\operatorname{Pr}[\neg \operatorname{RejlnvC}] \leq \mathbf{A d} \mathbf{v}_{\mathrm{H}}^{C O L}(\lambda)+\frac{q_{D}}{q},
$$

where $q_{D}$ denotes the number of the queries to the decapsulation oracle.

Proof of Claim 2. Suppose that $\mathcal{A}$ submits an invalid ciphertext $\psi=\left(u_{1}, u_{2}, v\right) \neq \psi^{*}$ to the decapsulation oracle. First, note that it is not possible that $\left(u_{1}, u_{2}\right)=\left(u_{1}^{*}, u_{2}^{*}\right)$ since $\psi \neq \psi^{*}$, we have $v \neq v^{*}$ and hence the decapsulation oracle will reject $\psi$ straight away. Note also that it is possible that $\left(u_{1}, u_{2}\right) \neq\left(u_{1}^{*}, u_{2}^{*}\right)$ and $\alpha=\alpha^{*}$ but the probability that this happens is bounded by the insecurity of the hash function $\mathrm{H}$ since this event implies the violation of the target collision-resistance of $\mathrm{H}$.

Thus, for up to $q_{D}$ invalid ciphertexts such that $\left(u_{1}, u_{2}\right) \neq\left(u_{1}^{*}, u_{2}^{*}\right)$, we have $\alpha \neq \alpha^{*}$. In this case, if the point $\left(x_{1}, x_{2}, y_{1}, y_{2}\right)$ lied on the hyperplane defined by the following equation

$$
\log _{g_{1}} v=r_{1} x_{1}+r_{2} x_{2} w+r_{1} y_{1} \alpha+r_{2} y_{2} w \alpha
$$

where $r_{1}=\log _{g_{1}} u_{1}$ and $r_{2}=\log _{g_{1}} u_{2}$, the decapsulation oracle would accept the ciphertext $\psi$. However, observe that

$$
\operatorname{det}\left[\begin{array}{cccc}
1 & w & 0 & 0 \\
0 & 0 & 1 & w \\
r_{1}^{*} & r_{2}^{*} w & r_{1}^{*} \alpha^{*} & r_{2}^{*} \alpha^{*} w \\
r_{1} & r_{2} w & r_{1} \alpha & r_{2} \alpha w
\end{array}\right]=w^{2}\left(r_{1}-r_{2}\right)\left(r_{1}^{*}-r_{2}^{*}\right)\left(\alpha^{*}-\alpha\right) \neq 0 .
$$

Hence, (2), (3), (10) and (14) are linearly independent, implying that the hyperplane defined by (14) intersects the line formed by intersecting (2), (3) and (10) at a point, which happens with negligible probability $1 / q$. Considering that there are $q_{D}$ decapsulation queries, we get (13). This is the end of proof of Claim 2.

Note that from (7) and (13), we get

$$
\begin{aligned}
\operatorname{Pr}\left[\beta^{\prime}=\beta\right] & =\operatorname{Pr}\left[\beta^{\prime}=\beta \mid \operatorname{Rej} \operatorname{lnv} C\right] \operatorname{Pr}[\operatorname{Rej} \operatorname{lnv} C]+\operatorname{Pr}\left[\beta^{\prime}=\beta \mid \neg \operatorname{Rej} \operatorname{lnv} C\right] \operatorname{Pr}[\neg \operatorname{RejInvC}] \\
& \leq \operatorname{Pr}\left[\beta^{\prime}=\beta \mid \operatorname{Rej} \operatorname{lnv} C\right]+\operatorname{Pr}[\neg \operatorname{Rej} \operatorname{lnv} C] \\
& \leq \frac{1}{2}+\operatorname{Adv}_{\mathrm{KDF}}^{\mathrm{ROR}}(\lambda)+\operatorname{Adv}_{\mathrm{H}}^{\mathrm{COL}}(\lambda)+\frac{q_{D}}{q} .
\end{aligned}
$$


(The above inequality shows that regardless of the quantity of $\operatorname{Pr}\left[\beta^{\prime}=\beta \mid \neg \operatorname{Rej} \operatorname{lnvC}\right]$, i.e. the advantage that the adversary may get through querying invalid ciphertexts to the decapsulation oracle, $\operatorname{Pr}\left[\beta^{\prime}=\beta\right]$ is not much deviated from $1 / 2$ due to $\operatorname{Pr}\left[\beta^{\prime}=\beta \mid \neg \operatorname{Rej} \operatorname{lnvC}\right]$ and $\operatorname{Pr}[\neg \operatorname{Rej} \mid n v C]$, which turn out to be negligible.)

Then, from the construction of $\mathcal{D}$, we have

$$
\operatorname{Pr}\left[\mathcal{D}\left(1^{\lambda}, g_{1}, g_{2}, g_{1}^{r_{1}^{*}}, g_{2}^{r_{2}^{*}}\right)=1\right]=\operatorname{Pr}\left[\beta^{\prime}=\beta\right] \leq \frac{1}{2}+\mathbf{A d v}_{\mathrm{KDF}}^{\mathrm{ROR}}(\lambda)+\operatorname{Ad}_{\mathbf{H}}^{\mathrm{COL}}(\lambda)+\frac{q_{D}}{q} .
$$

Combining the bounds from Lemmas 1 and 2 (i.e., by subtracting (1) from (6)), we get the bound in the theorem statement.

\section{Comparisons}

In Table 1, we summarize the basic parameters such as public key, ciphertext of CS-KEM [10], KD-KEM [17], HK-KEM [14] and ours. We also summarize whether those schemes provide full CCA-security, assuming the hardness of the DDH problem. Note that KD-KEM and HK-KEM are proven to be CCCA-secure [14], which is weaker than full CCA. Note also that it is an open problem to prove or disprove that HK-KEM provides full CCA-security.

\begin{tabular}{|l|l|l|l|l|}
\hline Scheme & Public key & Ciphertext & Key & Full CCA \\
\hline \hline CS-KEM [10] & $g_{1}, g_{2}, c, d, h$ & $g_{1}^{r}, g_{2}^{r},\left(c d^{\alpha}\right)^{r}$ & $\operatorname{KDF}\left(g_{1}^{r}, h^{r}\right)$ & Yes \\
\hline KD-KEM [17] & $g_{1}, g_{2}, c, d$ & $g_{1}^{r}, g_{2}^{r}$ & $\left(c d^{\alpha}\right)^{r}$ & No \\
\hline HK-KEM [14] & $g_{1}, c, d, h$ & $g_{1}^{r},\left(c d^{\alpha}\right)^{r}$ & $h^{r}$ & Not Known \\
\hline Ours & $g_{1}, g_{2}, c, d$ & $g_{1}^{r}, g_{2}^{r},\left(c d^{\alpha}\right)^{r}$ & $\operatorname{KDF}\left(g_{1}^{r}, c^{r}\right)$ & Yes \\
\hline
\end{tabular}

Table 1: Comparison of Our KEM Scheme with Other KEM Schemes

As one can notice from the above table, our scheme is more efficient than the CS-KEM scheme while it is less efficient than the KD-KEM and HK-KEM schemes. However, an advantage of our scheme and CS-KEM schemes might be the simplicity that they provide full CCA-security without introducing additional primitive like MAC. - As formally shown in [3], one can generically convert a CCCA-secure KEM into a CCA-secure KEM by authenticating the CCCA-secure KEM ciphertext using a MAC. Hence, KD-KEM and HK-KEM can be made to be CCA-secure by introducing the overhead of MAC. In this case, expansion of the ciphertext is unavoidable and as a result, the length of the ciphertext is close to the original CS-KEM and ours.

In Table 2, we summarize the computational costs of the above-mentioned schemes. In the table, "E" stands for "Exponentiation", "DE" stands for "Double Exponentiation", which is a special case of multi-exponentiation for two bases, e.g. $A^{a} B^{b}$, and finally "SE" stands for "Sequential Exponentiation" [7], which is as efficient as multi-exponentiation (in our case, double exponentiation).

Since there are many factors that determine the running time of various multi-exponentiation algorithms [2, 19], it would be difficult to state decisively one double exponentiation is equivalent to how much of single exponentiation. (Note that if we use the naive approach that 
computes two single exponentiations separately and multiply them together, $1 \mathrm{DE}=2 \mathrm{E}$.) But if one adopts the "multi-exponentiation with a sliding window" algorithm assuming the unsigned binary representation of exponents as described in [2], one can obtain $1 \mathrm{DE}=1.39 \mathrm{E}$ if window size $=2$ and the bit-length of $q=256$. The figures in the parentheses in Table 2 are obtained based on this assumption.

\begin{tabular}{|l|l|l|}
\hline Scheme & Enc. Cost & Dec. Cost \\
\hline \hline CS-KEM $[10]$ & $3 \mathrm{E}+1 \mathrm{DE}(4.39 \mathrm{E})$ & 2DE $(2.78 \mathrm{E})$ \\
\hline KD-KEM $[17]$ & $2 \mathrm{E}+1 \mathrm{DE}(3.39 \mathrm{E})$ & 1DE $(1.39 \mathrm{E})$ \\
\hline HK-KEM $[14]$ & $2 \mathrm{E}+1 \mathrm{DE}(3.39 \mathrm{E})$ & $1 \mathrm{SE}(\approx 1.39 \mathrm{E})$ \\
\hline Ours & $4 \mathrm{E}$ & $2.78 \mathrm{E}$ \\
\hline
\end{tabular}

Table 2: Comparison of Computational Costs

Notice from the above table that in terms of computational costs, the difference between our scheme and both KD-KEM and HK-KEM is less than one exponentiation.

We also remark that as done for CS-KEM and KD-KEM respectively in [10] and in [21], one can make the key generation and decapsulation algorithms of our KEM scheme more efficient, which is described in detail in Appendix A.

\section{Conclusion}

In this paper, we proposed a new variant of the Cramer-Shoup KEM (CS-KEM) scheme which is more efficient than the original Cramer-Shoup KEM and fully CCA-secure in the standard model, relative to the DDH problem. Our result shows that the original CS-KEM can further be optimized without losing full CCA-security.

It is natural to ask whether the same technique (that is, to "reuse" $d^{r}$ in $\left.\left(c^{\alpha} d\right)^{r}\right)$ can be applied to the dual version of KD-KEM scheme presented in [14]. We found that it is difficult to provide a security reduction in this case since, when an inconsistent ciphertext is queried to the decapsulation oracle, one cannot always extract a key uniformly distributed in the simulation.

Thus, an interesting open problem is how to construct a PKE scheme that is more efficient than the PKE schemes based on the KD-KEM or the dual KD-KEM.

\section{Acknowledgement}

The authors are grateful to Eike Kiltz for his suggestions of improvement on an earlier version of this paper.

\section{References}

[1] M. Abe, R. Genaro and K. Kurosawa, Tag-KEM/DEM: A New Framework for Hybrid Encryption and A New Analysis of Kurosawa-Desmedt KEM, Cryptology ePrint Archive, Report 2005/027, 2005. (Last update: 11 October 2006.) 
[2] R. M. Avanzi, The Complexity of Certain Multi-Exponentiation Techniques in Cryptography, Journal of Cryptology, Vol. 18 (4), pp. 357 - 373, Springer-Verlag, 2005.

[3] J. Baek, D. Galindo, W. Susilo and J. Zhou, Constructing Strong KEM from Weak KEM (or How to Revive the KEM/DEM Framework), Proc. of the 6th Conference on Security and Cryptography for Networks (SCN 2008), LNCS 5229, pp. 358-374, Springer-Verlag, 2008.

[4] M. Bellare, R. Canetti and H. Krawczyk, Keying Hash Functions for Message Authentication, In Crypto '96, LNCS 1109, pp. 1-15, Springer-Verlag, 1996.

[5] M. Bellare, A. Desai, D. Pointcheval and P. Rogaway, Relations Among Notions of Security for Public-Key Encryption Schemes, In Crypto '98, LNCS 1462, pp. 26-45, Springer-Verlag, 1998.

[6] M. Bellare and P. Rogaway, Random Oracles are Practical: A Paradigm for Designing Efficient Protocols, In ACM-CCS '93, pp. 62-73, ACM, 1993.

[7] D.J. Bernstein, Pippenger's Exponentiation Algorithm, Preprint, 2002. Available from http://cr.yp.to

[8] D. Cash, E. Kiltz and V. Shoup, The Twin Diffie-Hellman Problem and Applications, In Eurocrypt '08, LNCS 4965, pp. 127-145, Springer-Verlag, 2008. Full version available on Cryptology ePrint Archive: Report 2008/067

[9] R. Cramer and V. Shoup, A Practical Public Key Cryptosystem Provably Secure against Adaptive Chosen Ciphertext Attack, In Crypto '98, LNCS 1462, pp. 13-25, SpringerVerlag, 1998.

[10] R. Cramer and V. Shoup, Design and Analysis of Practical Public-key Encryption Schemes Secure against Adaptive Chosen Ciphertext Attack, SIAM Journal of Computing 33, pp. 167-226, 2003.

[11] D. Dolev, C. Dwork and M. Naor, Non-malleable Cryptography, In STOC '91, pp. 542552, ACM, 1991.

[12] R. Gennaro and V. Shoup, A Note on An Encryption Scheme of Kurosawa and Desmedt, Cryptology ePrint Archive, Report 2004/294, 2004.

[13] J. Herranz, D. Hofheinz and E. Kiltz, The Kurosawa-Desmedt Key Encapsulation is not Chosen-Ciphertext Secure,Cryptology ePrint Archive, Report 2006/207, 2006.

[14] D. Hofheinz and E. Kiltz. Secure Hybrid Encryption from Weakened Key Encapsulation, In LNCS 4622, CRYPTO 2007, pp. 553-571, Springer-Verlag, 2007.

[15] ISO 18033-2, An Emerging Standard for Public-Key Encryption, 2004.

[16] E. Kiltz, Chosen-Ciphertext Secure Key-Encapsulation Based on Gap Hashed DiffieHellman, In PKC '07, LNCS 4450, pp. 282-297, Springer-Verlag, 2007. 
[17] K. Kurosawa and Y. Desmedt, A New Paradigm of Hybrid Encryption Scheme, In Crypto '04, LNCS 3152, pp. 426-442, Springer-Verlag, 2004.

[18] X. Lu, X. Lai and D. He, Improved efficiency of Kiltz07-KEM, Cryptology ePrint Archive, Report 2008/312, 2008.

[19] B. Möller and A. Rupp, Faster Multi-Exponentiation through Caching: Accelerating (EC)DSA Signature Verification, In Security and Cryptography for Networks (SCN 2008), LNCS 5229, pp. 39-56, Springer-Verlag, 2008.

[20] M. Naor and M. Yung, Public-key Cryptosystems Provably Secure against Chosen Ciphertext Attacks, In STOC '90, pp. 427-437, ACM, 1990.

[21] L. T. Phong and W. Ogata, On Some Variations of Kurosawa-Desmedt Public-Key Encryption Scheme, IEICE Transactions 90-A(1), pp. 226-230, 2007.

[22] V. Shoup, A Proposal for an ISO Standard for Public Key Encryption (version 2.1), ISO/IEC JTC 1/SC 27, 2001.

\section{A An Efficient Variant of Our KEM Scheme}

Description. Adopting the techniques in [10, 21], one can design an efficient variant of our

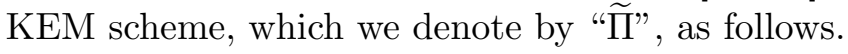

Key Generation: Pick a group $\mathbb{G}$ of prime order $q$ and generator $g_{1}$ of $\mathbb{G}$. Pick a target-collision resistant hash function $\mathrm{H}:\{0,1\}^{*} \rightarrow \mathbb{Z}_{q}^{*}$ and a key derivation function KDF. Then choose $(w, x, y) \in \mathbb{Z}_{q}^{3}$ at random and compute

$$
g_{2}=g_{1}^{w}, c=g_{1}^{x}, d=g_{1}^{y} .
$$

Return public key $p k=\left(\mathbb{G}, q, g_{1}, g_{2}, c, d, \mathrm{H}, \mathrm{KDF}\right)$ and private key $s k=(p k, x, y, w)$.

Encapsulation: Pick $r \in \mathbb{Z}_{q}^{*}$ at random. Compute

$$
u_{1}=g_{1}^{r}, u_{2}=g_{2}^{r}, \alpha=\mathrm{H}\left(u_{1}, u_{2}\right), v=c^{r} d^{r \alpha}, K=\operatorname{KDF}\left(u_{1}, c^{r}\right) .
$$

Return ciphertext $\psi=\left(u_{1}, u_{2}, v\right)$ and key $K$.

Decapsulation: Upon receiving $\psi=\left(u_{1}, u_{2}, v\right)$, compute

$$
\alpha=\mathrm{H}\left(u_{1}, u_{2}\right), u_{2}^{\prime}=u_{1}^{w}, v^{\prime}=u_{1}^{x+y \alpha}, K=\operatorname{KDF}\left(u_{1}, u_{1}^{x}\right) .
$$

If $u_{2}^{\prime}=u_{2}$ and $v^{\prime}=v$ then return $K$; otherwise, return $\perp$.

The above scheme is also CCA-secure. Regarding this, we prove the following theorem.

Theorem 2 If the KEM scheme $\Pi$ (described in Section 3) is CCA-secure then the above KEM scheme $\widetilde{\Pi}$ is CCA-secure. More precisely, we have

$$
\operatorname{Adv}_{\widetilde{\Pi}}^{C C A}(\lambda) \leq \operatorname{Adv}_{\Pi}^{C C A}(\lambda)+\frac{q_{D}}{q} .
$$

where $\lambda$ denotes the security parameter and $q_{D}$ is the number of queries to the decapsulation oracle. 
Proof. Fix an attacker $\mathcal{A}$ for the scheme $\Pi$. Also, fix an attacker $\tilde{\mathcal{A}}$ for the scheme $\widetilde{\Pi}$.

Assume that $\mathcal{A}$ is provided with the public key $p k=\left(\mathbb{G}, q, g_{1}, g_{2}, c, d\right)$ and the private key $s k=\left(p k, x_{1}, x_{2}, y_{1}, y_{2}\right)$, where $g_{1}$ and $g_{2}$ are generators of $\mathbb{G}$ and $c=g_{1}^{x_{1}} g_{2}^{x_{2}}$ and $d=g_{1}^{y_{1}} g_{2}^{y_{2}}$. $\mathcal{A}$ simply gives $\tilde{\mathcal{A}} p k$ as the public key of the scheme $\Pi$. $\mathcal{A}$ sets $g_{2}=g_{1}^{w}$ for some $w \in \mathbb{Z}_{q}$, $x=x_{1}+w x_{2}$ and $y=y_{1}+w y_{2}$. (Note that $\mathcal{A}$ does not the value $w$.) Since $c=g_{1}^{x_{1}} g_{2}^{x_{2}}=$ $g_{1}^{x_{1}+w x_{2}}=g^{x}, d=g_{1}^{y_{1}} g_{2}^{y_{2}}=g_{1}^{y_{1}+w y_{2}}=g^{x}$ by definition of $w$ and $(x, y)$, the public key $p k$ is distributed identically in both $\mathcal{A}$ and $\tilde{\mathcal{A}}$ 's view.

When $\tilde{\mathcal{A}}$ queries a ciphertext $\psi=\left(u_{1}, u_{2}, v\right)$ to the decapsulation oracle in the find stage, $\mathcal{A}$ forwards it to its decapsulation oracle, gets a decapsulation result and sends it back to $\tilde{\mathcal{A}}$.

Sometime later, $\mathcal{A}$ gets a challenge ciphertext and a key pair $\left(\psi^{*}\left(=u_{1}^{*}, u_{2}^{*}, v^{*}\right), K_{\beta}\right)$, where $\beta \in\{0,1\}$ is chosen at random, and forwards the pair to $\tilde{\mathcal{A}}$ as a challenge ciphertext of the scheme $\widetilde{\Pi}$ and a key.

When $\tilde{\mathcal{A}}$ queries a ciphertext $\psi=\left(u_{1}, u_{2}, v\right)$ to the decapsulation oracle in the guess stage, $\mathcal{A}$ forwards it to its decapsulation oracle, gets a decapsulation result and sends it back to $\tilde{\mathcal{A}}$.

When $\tilde{\mathcal{A}}$ outputs its guess, $\mathcal{A}$ outputs it as its guess.

We compute the probability that an invalid ciphertext $\psi=\left(u_{1}, u_{2}, v\right)$, which should have been rejected, is accepted by the simulated decapsulation oracle.

Since we have assumed that $\psi=\left(u_{1}, u_{2}, v\right)$ is invalid, the condition $\left[\left(u_{1}^{w} \neq u_{2}\right) \wedge\left(u_{1}^{x+y \alpha}=\right.\right.$ $v)]$ or $\left[\left(u_{1}^{w}=u_{2}\right) \wedge\left(u_{1}^{x+y \alpha} \neq v\right)\right]$ or $\left[\left(u_{1}^{w} \neq u_{2}\right) \wedge\left(u_{1}^{x+y \alpha} \neq v\right)\right]$ holds. However, if the last two conditions held, the simulated decapsulation oracle would reject $\psi$. Hence the first condition $\left[\left(u_{1}^{w} \neq u_{2}\right) \wedge\left(u_{1}^{x+y \alpha}=v\right)\right]$ must hold when invalid $\psi$ is not rejected by the simulated decapsulation oracle. Note that $u_{1}^{w} \neq u_{2}$ means $r_{1} \neq r_{2}$ where $r_{1}=\log _{g_{1}} u_{1}$ and $r_{2}=\log _{g_{1}} u_{2}$. Note also that since $x=x_{1}+w x_{2}$ and $y=x_{y}+w y_{2}, u_{1}^{x+y \alpha}=v$ is equivalent to

$$
\begin{aligned}
& {\left[r_{1}\left\{\left(x_{1}+w x_{2}\right)+\left(y_{1}+w y_{2}\right) \alpha\right\}\right] \bmod q } \\
= & {\left[r_{1}\left(x_{1}+y_{1} \alpha\right)+r_{2} w\left(x_{2}+y_{2} \alpha\right)\right] \bmod q } \\
\Longleftrightarrow & w\left(r_{1}-r_{2}\right)\left(x_{1}+w y_{2}\right)=0 \bmod q .
\end{aligned}
$$

As $r_{1} \neq r_{2}$ by the assumption and $w \neq 0 \bmod q$, the above equation holds with probability $1 / q$, which is negligible. Hence we get the bound in the theorem statement. 